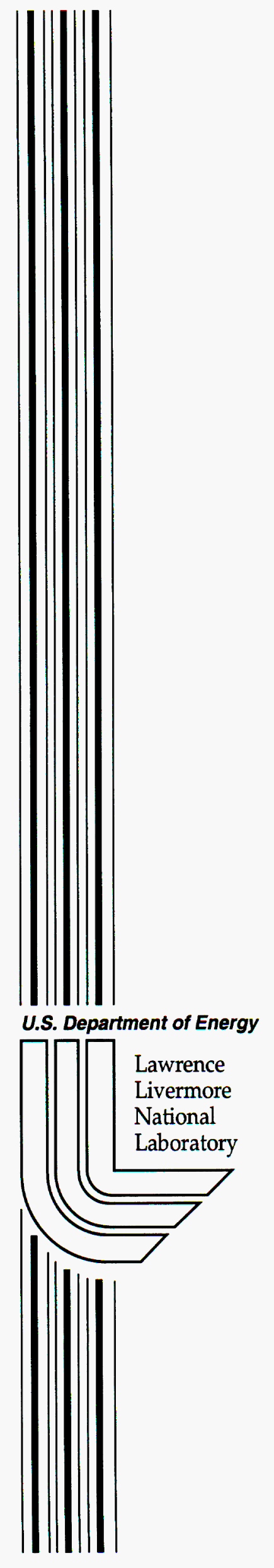

UCRL-ID-145157

\title{
Using Vulcan to Recreate Planetary Cores
}

G.W. Collins, P.M. Celliers, D.G. Hicks, A.J. Mackinnon, S.J. Moon, R. Cauble, L.B. DaSilva, M. Koenig, A. Benuzzi-Mounaix, G. Huser, R. Jeanloz, K.M. Lee, L.R. Benedetti, E. Henry, D. Batani, P. Loubeyre, O. Willi, J. Pasley, H. Gessner, D. Neely, M. Notley, C. Danson

\section{August 15, 2001}




\title{
DISCLAIMER
}

This document was prepared as an account of work sponsored by an agency of the United States Government. Neither the United States Government nor the University of California nor any of their employees, makes any warranty, express or implied, or assumes any legal liability or responsibility for the accuracy, completeness, or usefulness of any information, apparatus, product, or process disclosed, or represents that its use would not infringe privately owned rights. Reference herein to any specific commercial product, process, or service by trade name, trademark, manufacturer, or otherwise, does not necessarily constitute or imply its endorsement, recommendation, or favoring by the United States Government or the University of California. The views and opinions of authors expressed herein do not necessarily state or reflect those of the United States Government or the University of California, and shall not be used for advertising or product endorsement purposes.

This work was performed under the auspices of the U. S. Department of Energy by the University of California, Lawrence Livermore National Laboratory under Contract No. W-7405-Eng-48.

This report has been reproduced directly from the best available copy.

Available electronically at http://www.doe.gov/bridge

\author{
Available for a processing fee to U.S. Department of Energy \\ and its contractors in paper from \\ U.S. Department of Energy \\ Office of Scientific and Technical Information \\ P.O. Box 62 \\ Oak Ridge, TN 37831-0062 \\ Telephone: (865) 576-8401 \\ Facsimile: (865) 576-5728 \\ E-mail: reports@adonis.osti.gov \\ Available for the sale to the public from \\ U.S. Department of Commerce \\ National Technical Information Service \\ 5285 Port Royal Road \\ Springfield, VA 22161 \\ Telephone: (800) 553-6847 \\ Facsimile: (703) 605-6900 \\ E-mail: orders@ntis.fedworld.gov \\ Online ordering: http://www.ntis.gov/ordering.htm
}

\section{OR}

Lawrence Livermore National Laboratory

Technical Information Department's Digital Library

http://www.llnl.gov/tid/Library.html 


\title{
Using Vulcan to Recreate Planetary Cores
}

\author{
G. W. Collins, P. M. Celliers, D. G. Hicks, A. J. Mackinnon, S. J. Moon, R. Cauble, L. B. DaSilva \\ Lawrence Livermore National Laboratory, Livermore CA 94550 USA
}

\author{
M. Koenig, A. Benuzzi-Mounaix, G. Huser \\ LULI, Ecole Polytechnique, France
}

R. Jeanloz, K. M. Lee, L. R. Benedetti

University of California, Berkeley, U. S. A.

E. Henry, D. Batani

Univ. Milan, Italy

P. Loubeyre

CEA, France

O. Willi, J. Pasley, H. Gessner

Imperial College, London, U. K.

D. Neely, M. Notley, C. Danson

Central Laser Facility, CLRC Rutherford Appleton Laboratory, Chilton, Didcot, Oxon., OX11 OQX

Main contact email address collins7@llnl.gov

\section{Introduction}

An accurate equation of state (EOS) for planetary constituents at extreme conditions is the key to any credible model of planets or low mass stars. However, experimental validation has been carried out on at high pressure (>few Mbar), and then only on the principal Hugoniot. For planetary and stellar interiors, compression occurs from gravitational force so that material states follow a line of isentropic compression (ignoring phase separation) to ultra-high densities ${ }^{1}$.

An example of the predicted states for water along the isentrope for Neptune is shown in Fig. 1. The cutaway figure on the left is from Hubbard ${ }^{2}$, and the phase diagram on the right is from
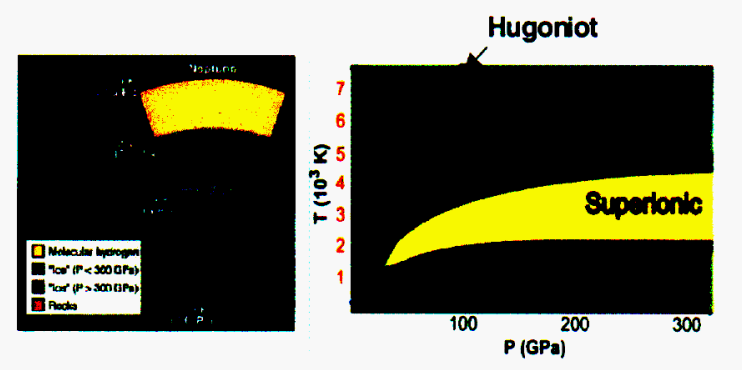

Fig. 1. Calculated equation of state of water from Ref. 3 and predicted cutaway of Neptune from Ref. 2.

Cavazzoni et al. ${ }^{3}$ Clearly these states lie at quite a bit lower temperature and higher density than single shock Hugoniot states but they are at higher temperature than can be achieved with accurate diamond anvil experiments.

At extreme densities, material states are predicted to have quite unearthly properties such as high temperature superconductivity ${ }^{4}$ and low temperature fusion ${ }^{5}$. High density experiments on Earth are achieved with either static compression techniques (i.e.diamond anvil cells) or dynamic compression techniques using large laser facilities, gas guns, or explosives. A major thrust of this work is to develop techniques to create and characterize material states that exists primarily at the core of giant planets and brown dwarf stars.

Typically, models used to construct planetary isentropes are constrained by only the planet radius, outer atmospheric spectroscopy, and space probe gravitational moment and magnetic field data. Thus any data, which provide rigid constraints for these models will have a significant impact on a broad community of planetary and condensed matter scientists.

Recent laser shock wave experiments have made great strides in recreating material states that exist in the outer $25 \%$ (in radius) of the Jovian planets and at the exterior of low-mass stars. Large laser facilities have been used to compressed materials to ultra-high pressures and characterize their thermodynamic and transport properties (plastic Hugoniot to $40 \mathrm{Mbar}$, deuterium Hugoniot to $3 \mathrm{Mbar}$, metallization of "atomic" deuterium on the Hugoniot). To probe materials properties at these high pressures, several experimental techniques were developed: high resolution radiography, optical reflectance, pyrometry, and velocity/displacement sensitive interferometry are some of the diagnostics currently used in laser-generated shock EOS experiments.

During our experiments at Vulcan we developed and tested precompressed and multiple shock experimental techniques which allowed us to recreate the extreme core states of giant plants. These experiments compressed water to densities higher than accessible by single shock Hugoniot techniques and showed that the metal-insulator transition of shocked precompressed water is suppressed significantly as compared to uncompressed water. Further, as predicted the temperature of shocked precompressed water is lower than the temperature of uncompressed water enabling us to determine the metallization mechanism for water near the Hugoniot.

\section{Experimental summary}

The double shock target design is sketched in Fig. 2. For these experiments, the Vulcan laser was used to ablate $\mathrm{CH}$, launching a shock into an $\mathrm{Al}$ step, which unloaded and launched a shock into water or other planetary fluid. Either a 1 ns or 4 ns pulse is used to launch a decaying or steady shock wave respectively. Typically reflectivity can be determined with decaying shocks while Hugoniot data require steady shocks. Rutherford Appleton Laboratory's (RAL) Vulcan Laser in target Area East generated drive pulses of $10^{13}-10^{14} \mathrm{~W} / \mathrm{cm}^{2}$ for 1 to $4 \mathrm{~ns}$ and with a $400 \mu \mathrm{m}$ diameter footprint. The footprint was spatially smoothed with PZP phase plates built at RAL. In these experiments, Vulcan was configured with six $108 \mathrm{~mm}$ diameter beams arranged in a hexagonal pattern with each beam incident on the target at 16 degrees from the normal and one additional $150 \mathrm{~mm}$ diameter beam at normal incidence. The $4 \mathrm{~ns}$ pulse was constructed by temporally staggering four sets of 1 ns pulses from the 7 beams. The $150 \mathrm{~mm}$ on axis beam arrived first, 
followed by pairs of the $108 \mathrm{~mm}$ beams that were timed to arrive at 1 ns intervals. The temporal dependence of the pulseshape was measured insitu with a pulseshape monitor.

We generated reflected shock states by reflecting shocks from a fused silica anvil. A sketch of the target design is shown in Fig. 2.

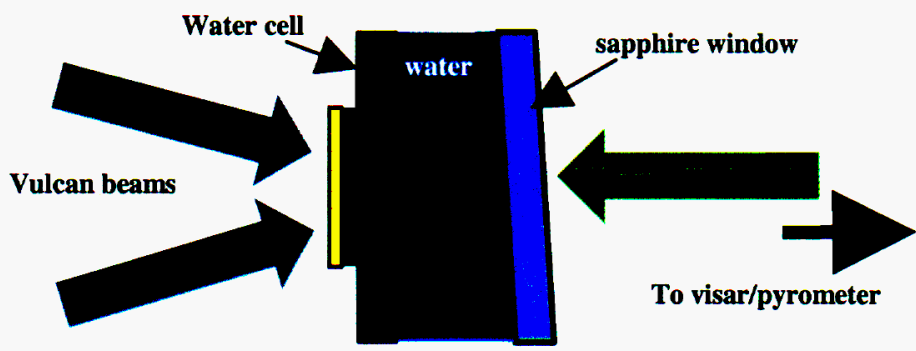

Fig. 2 Double shock water cell and experimental configuration.

The water shock transits the gap between the thin Al step and the $\mathrm{SiO}_{2}$ anvil, and then reflects off of the higher impedance $\mathrm{SiO}_{2}$ anvil.

\section{Diagnostics}

A VISAR (velocity interferometric system for any reflector) diagnostic was used to measure the shock velocity in the water versus time and the average shock velocity in the aluminum across the step. ${ }^{6}$ These kinematic observables and the known $\mathrm{Al}$ EOS were used to determine the equation of state of water. This diagnostic reflects a probe beam (532 nm $15 \mathrm{~ns})$ off the rear of the target, and relays an image of the target in the reflected beam through an interferometer and onto a streak camera. In most of these cases, after the shock breaks out of the aluminum pusher, light is reflected directly off of the water shock front. The Doppler shift of the light reflected from the shock front, is manifested as a fringe shift at the output of the velocity interferometer. The fringe phase, $\phi$, (in radians) produced by the velocity interferometer is related to the velocity, $u$, of the reflecting surface through the velocity interferometer equation $u=\lambda \phi / 4 \pi m(1+\delta)$, where $\lambda$ is the laser wavelength in vacuum, $\mathrm{n}$ is the refractive index of the unshocked water, $\tau=d / c$ is the delay in the velocity interferometer ( $c$ is the speed of light), and $\delta$ is a wavelength dependent correction due to dispersion in the etalon. ${ }^{7}$ Depending on the experiment we used $3 \mathrm{~mm}$ and $7 \mathrm{~mm}$ etalons which produced 16.2 , and 37.4 ps delay yielding 15.9 , and 6.89 $\mu \mathrm{m} / \mathrm{ns} /$ fringe in a vacuum respectively. The precision of this measurement is determined by the accuracy in determining $\lambda$, $\mathrm{n}, \tau$, and $\phi$. Important to note is that this determination is independent of the sweep calibration in the recording instrumentation, and independent of target design and/or metrology. Since we can determine $\lambda, \mathrm{n}$ and $\tau$ to a precision of 4 significant figures, the main contribution to experimental error arises in extracting $\phi$ from the data. The shock velocity in $\mathrm{Al}$ is determined from the measured breakout time from each step and the measured step thickness.

An example data record is shown in Fig. 3. Before 2.7 ns the stationary fringes are produced from the reflection of the probe beam off the stationary aluminum pusher. At $\sim 2.8 \mathrm{~ns}$ ( $3.7 \mathrm{~ns}$ ) the shock breaks out from the thin (thick) aluminum step, launching a strong shock into the water. As we show below, the reflection shown in Fig. 3 after shock breakout from the Al step, is caused from light reflecting off the shock front in water. Also shown in Fig. 3 are several lineouts were we have converted phase into velocity.
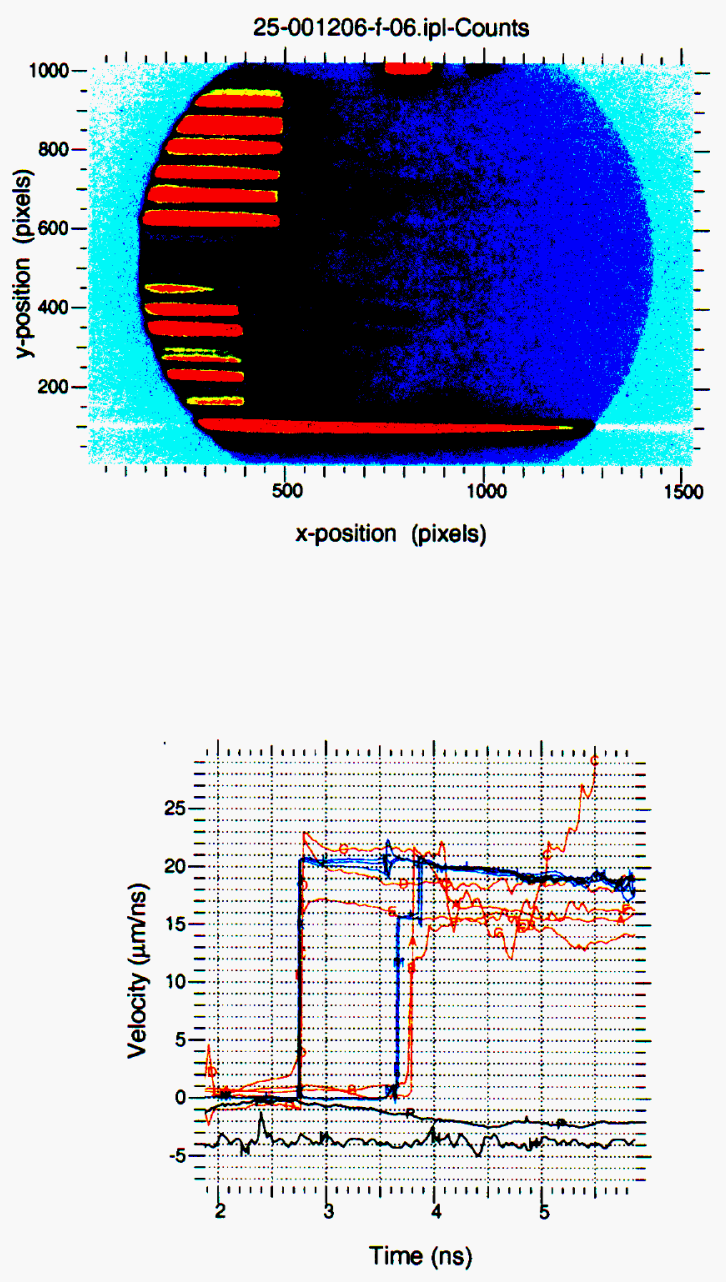

Fig. 3. Example data record from double shocked water experiment.

Equation of state measurements are typically presented as pressure versus density. To determine the shock pressure and density from shock velocities we use the RankineHugoniot relations; $P=\rho_{o} U_{s} U_{p}$ and $\rho=\rho_{o} U_{\mathcal{J}}\left(U_{s}-U_{p}\right)$, where $\rho_{o}$ is the initial density, $P$ is the shock pressure, and $U p$ is the particle velocity. We determine $U p$ (water) by standard impedance matching techniques with aluminum as the standard.

In addition to kinematic parameters, the VISAR diagnostic provides data to estimate the optical properties of shock compressed dielectrics. In the image shown above, (Fig. 3) the strong shock in water reflects light so that we track the shock front velocity and can estimate the reflectance of the shock front. In the case of weaker shocks, the shocked material can be opaque or transparent depending on the shock intensity and intrinsic properties of the material.

Temperature, is an important thermodynamic parameter but cannot be determined from the Rankine-Hugoniot equations. Temperature must be measured independently from shock and particle velocities. Typically, temperature of transparent shocked materials is measured from the thermal emission. We extracted $T$ by determining the spectral radiance, $I(\lambda)$ for a gray body Planck spectrum,

$$
I(\lambda)=\varepsilon(\lambda) \frac{2 \pi h c^{2}}{\lambda^{5}}\left(\exp \frac{h c}{\lambda k_{b} T}-1\right)^{-1}
$$


with $T$ as a fit parameter, $c, h$, and $k_{b}$ taking on their usual definitions, and the emissivity, $\varepsilon(\lambda)$, estimated from recent multi-wavelength reflectivity data described below.

To determine the spectral radiance from the sample we calibrated the transmission of our optics using a calibrated gray body source and the sensitivity of the streak camera by determining the response to a known intensity of a pulsed laser. An example data record for a double shock target is shown in Fig. 4. The temperature plotted in Fig. 4 assumes an emissivity of 1 . This is clearly not the case. To estimate the emissivity and determine the correct shock temperature we use the reflectance data described above, and use minimum conductivity model to estimate the collision time and then a Drude model is used to estimate carrier concentration. We then calculate the emissivity using a Drude model with these parameters evaluated at $610 \mathrm{~nm}$.

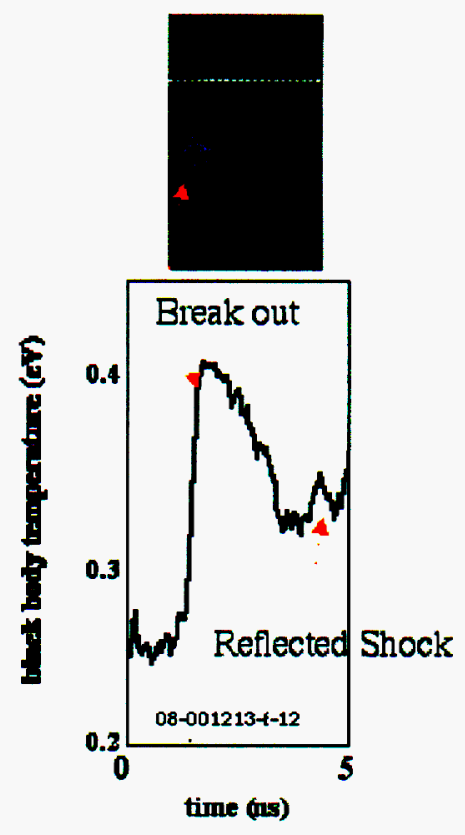

Fig. 4. Shock emission at $610 \mathrm{~nm}$ in water contained in a double shock cell.

\section{Precompressed targets}

A novel combination of the laser-driven shock technique with diamond anvil cell compression techniques is shown in Figure 5. With this target we used diamond flats to maintain a precompressed volume of our sample material at a static pressure of up to $1 \mathrm{GPa}$ during the experiment. The laser driver geometry described above results in two significant design constraints for achieving Hugoniot data on pre-compressed samples. First, the intensity and short pulse length require that the anvil through which the laser shock travels be extremely thin-no more than $200 \mu \mathrm{m}$. To ensure jump conditions are valid the shock must be steady. In order to have a steady shock wave persist into the sample with a square 4 ns pulse, the shock wave must propagate through the anvil and sample before the end-of-pulse rarefaction catches up. In addition, the shock planarity needs to be adequate for accurate inspection of the shock velocity. Thus, the spot size of the laser must be large enough so that side rarefactions do not erode the shock planarity to more than $\sim 75 \mu \mathrm{m}$. Second, the laser spot size and beam path require a large aperture radius (hence unsupported anvil radius), $r$, and aperture angle, $\theta$, in the high-pressure cell itself (Fig. 5).

In order to satisfy the needs for a thin anvil having a large unsupported aperture, we chose the strongest material known, diamond. Use of a diamond anvil cell is well established and the sample can be probed before and during the experiment due to the optical transparency of the anvil. Hence, the thickness, pressure and other characteristics of the pre-compressed sample can be determined before the Hugoniot experiment.

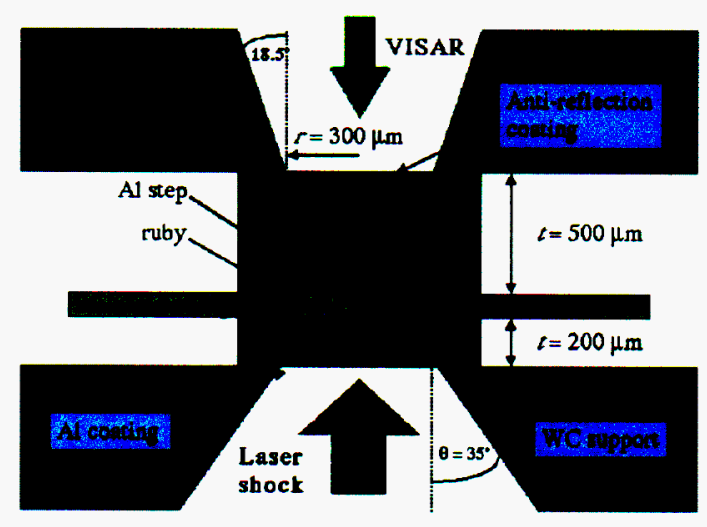

FIGURE 5. Schematic cross-section of diamond-cell configuration used for laser-driven shock experiments on precompressed samples. Wide openings ( $300 \mu \mathrm{m}$ radius holes) in tungsten carbide (WC) supports allow ample shock laser entry $\left(\theta=35^{\circ}\right.$ opening) and VISAR access (18.5 opening). Thin diamonds are pushed together to apply pressure on a small sample of water $(\sim 30 \mathrm{~nL})$ held in a hole within a stainless steel gasket $100 \mu \mathrm{m}$ thick. An Al step is glued on the thinnest diamond and used to measure the breakout times (velocities, and ultimately shock pressure) with VISAR. ${ }^{7}$ A few ruby grains are placed in the sample chamber for precompression pressure measurements via ruby fluorescence. ${ }^{8}$ There is a $1000 \AA \mathrm{Al}$ flash coating on the rear side of the thinnest diamond to lower the critical depth of shock ablation, and an anti-reflection coating on the thicker diamond for the VISAR measurement.

Plasma generated by ablation of the backing plate is a source of high energy $x$-rays that can preheat the sample. Also, the blowoff plasma absorbs the laser beam far from the target reducing the shock intensity significantly. In order to avoid preheat, plasma blow-off and laser damage to the DAC, a wide cone angle $\left(\theta=35^{\circ}\right)$ and diamond support hole ( $300 \mu \mathrm{m}$ radius) were incorporated into the design of the support plate for the diamond as well as the surrounding diamond cell. This geometry, although accommodating to the laser shock, provides less support for the anvil and is therefore less than ideal for the thin diamond flats.

\section{Characteristics of Thin Diamond Anvils}

The diamond flat can be modeled as a uniformly-loaded circular plate, such that a simple relation exists between the maximum pressure load $w$ and the anvil thickness $t$,

$$
w=\frac{k_{1} S_{m} r^{2}}{t^{2}}
$$

where $S_{m}$ is the maximum stress achieved in the diamond (here, the tensile strength of diamond), $r$ is the unsupported radius and $k_{l}$ is a constant equal to 0.833 (simply supported disc) or 1.333 (disc with fixed edges). ${ }^{9}$ For $r=300 \mu \mathrm{m}$ and $t=200 \mu \mathrm{m}$ and using the value of tensile strength of diamond, $2.8 \mathrm{GPa}(4)$, we arrive at a maximum load of between 1.0 (simply supported) and $1.7 \mathrm{GPa}$ (fixed edges)

Determining the initial pressure-density-internal energy conditions $\left(P_{0}, \rho_{0}, E_{0}\right)$ in the pre-compressed sample is key for Hugoniot measurements. The precompression pressure $P_{0}$ was measured in the water via ruby fluorescence ${ }^{8}$. Using the equation of state of water by Saul and Wagner, ${ }^{10}$ the initial density $\rho_{o}$ and energy $E_{o}$ were determined. 
White-light interferometry was used to determine the product of the index of refraction and distance $n d .^{11}$ The height of the Al step (known a priori) placed into the sample to aid in the VISAR measurements was used as $d$; determining the index of refraction for the compressed water sample was therefore straightforward (Fig. 6).

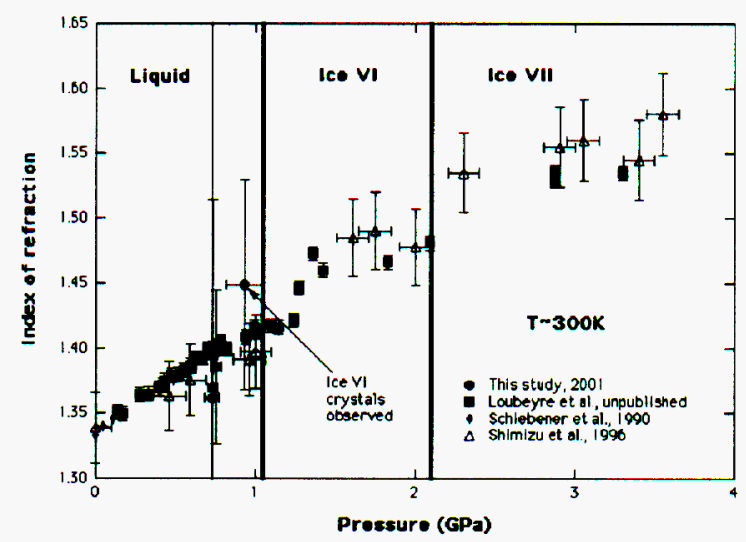

FIGURE 6. Index of refraction as a function of pressure for $\mathrm{H}_{2} \mathrm{O}$ water, ice VI and ice VII at room temperature. The values obtained in the present study (closed circles) compare well with previous results of Loubeyre et al., ${ }^{12}$ (closed squares) (7); Schiebener et al., (closed diamonds) $;^{13}$ and Shimizu et al., (open triangles). ${ }^{14}$ In one run, ice VI crystals were observed at a pressure less than the nominal freezing pressure, indicating a possibly metastable condition. ${ }^{15}$

Despite the fragility of the diamond flats, it was possible to precompress water to $\sim 1 \mathrm{GPa}\left(\rho_{0} \sim 1.2 \mathrm{~g} / \mathrm{cm}^{3}\right)$, as predicted by the above models. These precompressed samples were lasershocked up to pressures of $\sim 200 \mathrm{GPa}$ and temperatures to $\sim 10,000 \mathrm{~K}$ (see 11,12 ). In order to achieve higher initial pressures (and therefore densities), the laser intensity and duration must be increased to allow for thicker diamonds flats. For instance, the maximum load that a $500 \mu \mathrm{m}$ thick diamond flat could withstand is predicted to lie between 6.5 (simplysupported) and $12.5 \mathrm{GPa}$ (fixed-at-edges).

\section{Results and Discussion}

To test our experimental setup we compared single shock water EOS data to that collected on the Omega laser facility. The result shown in Fig. 7, reveals good agreement between data collected at Omega and Vulcan. The double shock data reached the highest pressure off Hugoniot values. However, for this discussion we will focus on the results of the precompressed experiments.

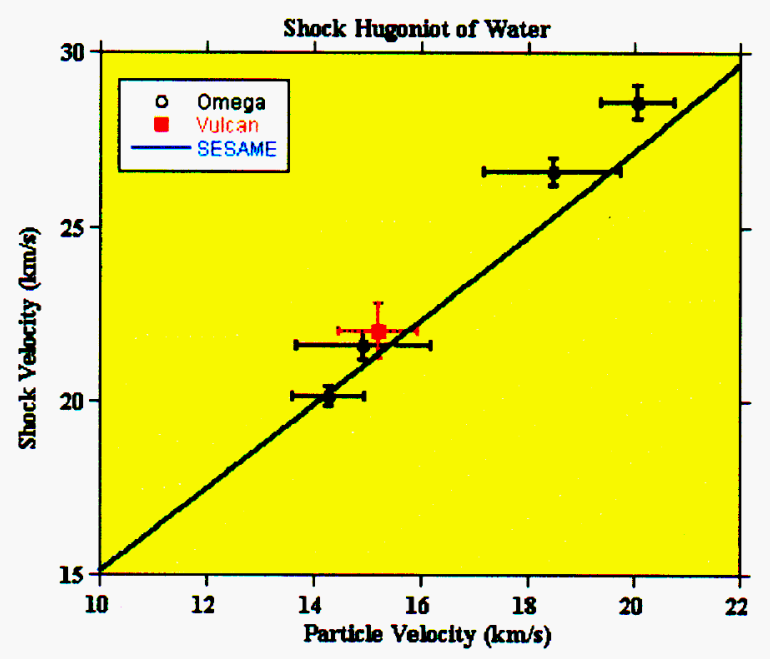

Fig. 7 Shock velocity versus particle velocity for water impedance matched to aluminum.

Four successful shots were achieved on pre-compressed targets: the highest pressure shot produced a reflecting shock, the lowest pressure produced shock-compressed material that remained transparent, and the two in between shocked the material into an opaque state, thus straddling the insulator-conducting transition and allowing a comparison with the principal Hugoniot states. All targets were pre-compressed to between $0.7-0.95 \mathrm{GPa}$, giving initial densities of $1.2 \mathrm{~g} / \mathrm{cm}^{3}, 20 \%$ above liquid density, and a refractive index of 1.4. An example precompressed VISAR data record is shown in Fig. 8.

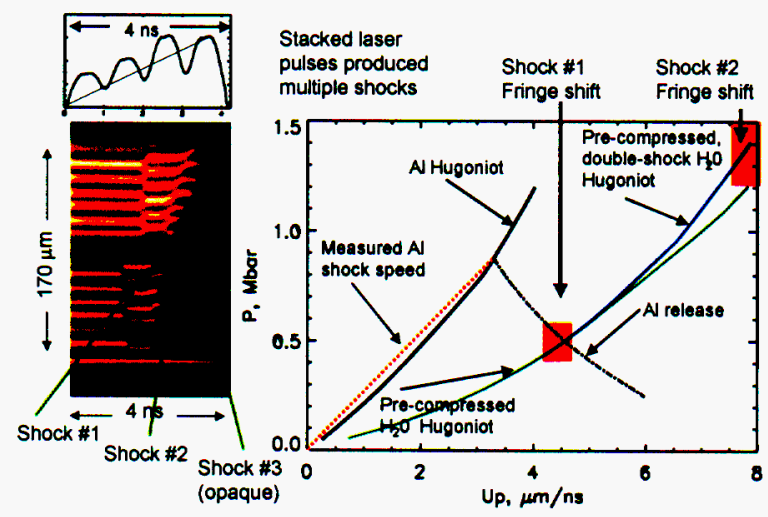

Fig. 8. Example precompressed water VISAR data record and analysis.

Shock pressures were determined by (1) the measured break-out times in the aluminum step together with the known step height yield the shock velocity in the $\mathrm{Al},(2)$ the shock velocity in the water from the VISAR measurements, and (3) impedance matching provided a model-based result for the state of shocked water. On shots where VISAR fringes could be observed either from the reflective water shock front, or from the moving aluminum interface, the pressure could also be determined directly using impedance matching with the known aluminum EOS. These model-based and direct techniques were in agreement for the transparent and reflecting shock states. For the opaque regime, a shock temperature was found for a single shot. This measured temperature was in agreement with the model-based temperature derived from impedance matching to 
the aluminum shock speed. Within the range explored by this experiment, it thus appears that the SESAME EOS for water is in agreement with experiment.

The highest pressure shot at $\sim 4$ Mbar produced a weakly reflecting shock. While the shock reflectivity could not be precisely determined due to the curvature of the shock front, it appeared clear that the reflectivity was significantly less than the $50 \%$ value found on the principal Hugoniot at the same pressure.

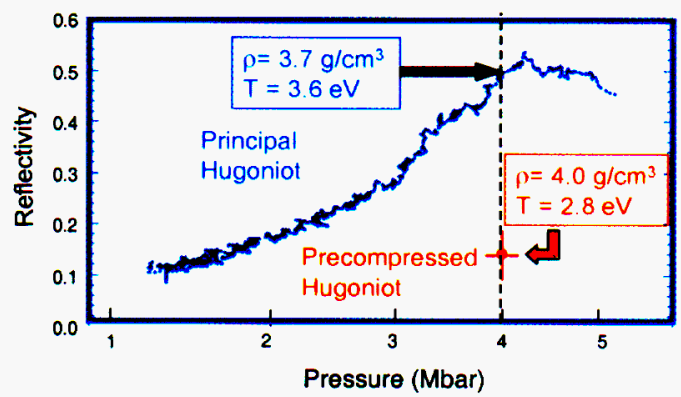

Fig. 8. Reflectivity from principal Hugoniot and precompressed Hugoniot experiments versus pressure.

Pre-compression thus appears to reduce the electronic conductivity. This sheds some light on the mechanism behind the insulator-conductor transition in water. At $4 \mathrm{Mbar}$, SESAME calculations indicate that the density and temperature are $3.7 \mathrm{~g} / \mathrm{cm}^{3}$ and $3.6 \mathrm{eV}$ on the principal Hugoniot and 4.0 $\mathrm{g} / \mathrm{cm}^{3}$ and $2.8 \mathrm{eV}$ in this pre-compressed shot. If the metalinsulator transition were due to a Mott transition, the higher density in the pre-compressed targets should increase the electronic conductivity of water rather than decrease it. On the other hand, if the transition were due to thermally-induced electron excitations, the lower temperature of the precompressed targets would reduce the reflectivity of the shock, as in fact these results show. Our experiments thus provide evidence that the insulator-conductor transition on the principal Hugoniot in water is thermally induced.

At the lowest pressure accessed, a shock of $0.5 \mathrm{Mbar}$ was shown to be transparent to the VISAR probe. This contrasts with water states on the principal Hugoniot, which were found to be opaque above $0.35 \mathrm{Mbar}$. Such a delayed onset of the transparent-opaque transition in pre-compressed water is consistent with the observation given above that the lower temperatures achieved in pre-compressed water reduce chargecarrier excitation.

An interesting observation in this shot was the effect of multiple shock states. The multiple shocks arose accidentally from the arrangement of stacked laser pulses. In order to produce the 4 ns drive pulse, it was necessary to arrange the seven $1 \mathrm{~ns}$ beams in sequence. However, early in the campaign, the pulse widths had not been optimized and gaps still existed between each nanosecond of the $4 \mathrm{~ns}$ drive. On this particular shot, the later pulses were stronger than earlier ones, leading to a sequence of shocks of increasing strength that converged in time. The VISAR streak shows these shocks as successive fringe shifts. The initial transparent state at $0.5 \mathrm{Mbar}$ is described above and is confirmed by both SESAME calculations and measured fringe shifts. ${ }^{16}$ The second shock state can only be inferred from the fringe shift, and is found to be about 1.4 Mbar, based on SESAME. The third shock accesses the opaque state of water at some unknown, higher pressure. The striking result of this shot is that a double-shocked state of pre-compressed water is found to be transparent up to $1.4 \mathrm{Mbar}$, with a corresponding temperature of $0.3 \mathrm{eV}$. Combining these results, the twodimensional P-T diagram of water can begin to be mapped out in terms of the regions of transparent, opaque, and reflecting shocks as shown in Fig. 9. Even modest pre-compression of samples can lead to important extensions in our understanding of material properties.

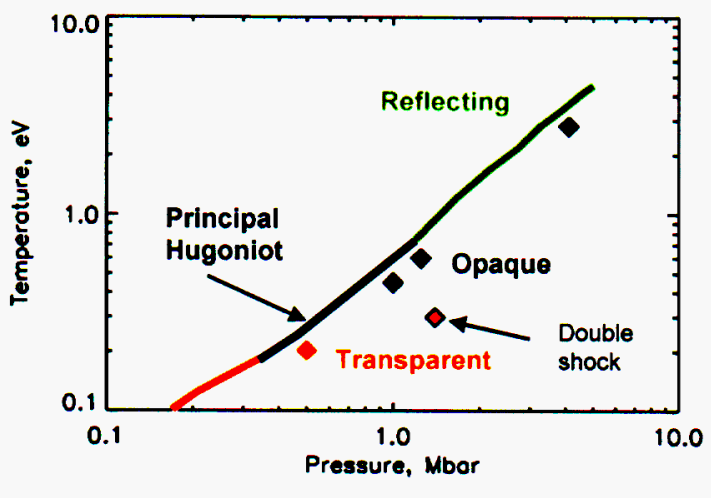

Fig. 9. Summary of the optical properties along the principal Hugoniot (line) and precompressed experiments (diamonds) in the T-P plane.

${ }^{1}$ Hubbard, W. B. Science 214, 145(1981). Hubbard, W. B. Guillot, T., Lunine, J. I., Burrows, A. Saumon, D., Marley, M. S., \& Freedman, R. S. 1997, Phys. Plasmas 4, 2011

${ }^{2}$ W. B. Hubbard, Science 214, 145 (1981).

${ }^{3}$ Cavazzoni et al, Science 284, 788 (1999).

${ }^{4}$ N. W. Ashcroft, Phys. World 8, 43 (July, 1995).

${ }^{5}$ S. Ishimaru, Rev. Mod. Phys. 65, 255 (1993).

${ }^{6}$ P.M.Celliers et al, Appl. Phys. Lett. 73, 1320 (1998).

${ }^{7}$ L. M. Barker and R. E. Hollenbach, J. Appl. Phys., 43, 4669-4675 (1972); L. M. Barker and K. W. Schuler, J. Appl. Phys., 45, 3692-3 (1974).

${ }^{8}$ Mao, H. K. et al., J. Appl. Phys. 49, 3276-3283 (1978).

${ }^{9}$ Timoshenko, S., Strength of Materials, Part II: Advanced

Theory and Problems, D. Van Nostrand Company, Princeton, New Jersey, 1956, pp. 76-144.

${ }^{10}$ Saul, A. and Wagner, W., J. Phys. Chem. Ref. Data 18, $1537-$ 564 (1989)

${ }^{11}$ Le Toullec, R. et al., Phys. Rev. B 40, 2368-2378 (1989).

12 Loubeyre, P. et al., unpublished (2000).

${ }^{13}$ Schiebener, P. et al., J. Phys. Chem. Ref. Data 19, 677-717 (1990).

${ }^{14}$ Shimizu, H. et al., Phys. Rev. B 53, 6107-6110 (1996).

15 Tkachev, S. et al., J. Chem. Phys. 105, 3722-3725 (1996).

${ }^{16}$ Note that in the case of transparent shocks, the fringe shifts correspond to the particle velocity of the shocked water, since the reflection occurs at the aluminum-water interface.

Consequently the refractive index of shocked water needs to be taken into account. Based on diamond cell measurements of water at similar densities, it is found that the refractive index of water almost follows Gladstone-Dale scaling, which means that the corrections to the inferred particle velocity are small. In this case they are less than $5 \%$. It is thus reasonable to assume that uncertainties in the inferred fringe shift due to unknown refractive index effects are in fact small. 